\title{
РЕЗУЛЬТАТЫ МОДИФИЦИРОВАННОЙ РЕВЕРСИВНОЙ ПЛАСТИКИ С ИСПОЛЬЗОВАНИЕМ ЛОСКУТА ЛЕВОЙ ПОДКЛЮЧИЧНОЙ АРТЕРИИ У ПАЦИЕНТОВ ПРИ КОАРКТАЦИИ АОРТЫ
}

\author{
Ю. С. Синельников ${ }^{1}$, Э. Н. Гасанов ${ }^{2}$, Ф. А. Мирзазаде ${ }^{2}$, И. А. Сойнов ${ }^{3}$
}

${ }^{1}$ Федеральный центр сердечно-сосудистой хирургии имени С. Г. Суханова МЗ РФ, г. Пермь, Россия,

${ }^{2}$ Научный центр хирургии имени М. А. Топчибашева, г. Баку, Республика Азербайджан,

${ }^{3}$ Национальный медицинский исследовательский центр имени академика Е. Н. Мешалкина МЗ РФ, г. Новосибирск, Россия

\section{RESULTS OF MODIFIED REVERSED PLASTY, USING THE FLAP OF THE LEFT SUBCLAVIAN ARTERY IN PATIENTS WITH AORTAL COARCTATION}

\author{
Yu. S. Sinelnikov', E. N. Gasanov², F. A. Mirzazade², I. A. Soynov³ \\ ${ }^{1}$ Sukhanov Federal Centre of Cardio-vascular Surgery, Perm, Russia, \\ ${ }^{2}$ Topchibashev Scientific Centre of Surgery, Baku, Republic of Azerbaijan, \\ ${ }^{3}$ Meshalkin National Medical Research Center, Novosibirsk, Russia
}

\section{Рефрерат}

Вступление. Коарктация аорты (КА) составляет от 5 до 8\% всех врожденных пороков сердца, часто ассоциируется с другими врожденными аномалиями, в частности, гипоплазией дуги аорты (ДА). Непосредственные результаты лечения больных значительно улучшены, однако отдаленные результаты нельзя назвать удовлетворительными. Клинические проблемы после коррекции КА включают повторную КА, персистирующую артериальную гипертензию, аневризму аорты и цереброваскулярных сосудов, раннюю ишемическую болезнь сердца. Высокую частоту осложнений связывают с выбранной методикой коррекции КА и вариантом формирования анастомоза. В настоящее время нет проспективных рандомизированных исследований, однозначно доказывающих преимущества того или иного способа коррекции КА с гипоплазией дистальной части ДА.

Целью проспективного исследования было сравнение двух методов: модифицированной реверсивной пластики (I група) и фрормирования «extended» анастомоза (II група).

Материалы и методы. Приведены результаты оценки хирургического лечения 64 пациентов раннего возраста по поводу КА в сочетании с гипоплазией дистальной части ДА в период с 2011 по 2015 г.

Результаты. Анализ отдаленных результатов проведен у $62(98,8 \%)$ пациентов в сроки до 48 мес. Совокупный показатель выживаемости в сроки до 4 лет составил 95,9\% - в I группе, 95,6\% - во II группе. Диаметр перешейка аорты у пациентов II группь в отдаленном периоде составлял $(0,98 \pm 0,4)$ мм, I группы - $(1,2 \pm 0,86)$ мм. В I группе повторной КА в отдаленном периоде не было у $98,2 \%$ пациентов, во II группе - у $96,3 \%$.

Выводы. Метод модифицированной реверсивной пластики с использованием лоскута левой подключичной артерии воспроизводимый и безопасный, о чем свидетельствовало отсутствие фатальных осложнений как в раннем, так и отдаленном периоде после выполнения вмешательства.

Ключевые слова: коарктация аорты; гипоплазия дуги аорты; хирургическая коррекция; повторная коарктация аорты.

Abstract

Introduction. Aortal coarctation (AC) constitutes $5-8 \%$ of the inborn heart failures, it is associated with other inborn anomalies including the aortal arch (AA) hypoplasia. Immediate results of treatment of patients are improved significantly, but remote results may not be considered satisfactory. Clinical problems after the AC correction include reoperative correction of AC, persisting arterial hypertension, aortal and the cerebrovascular vessels aneurysm, early ischemic heart disease. High rate of complications is connected with the chosen method of correction of AC and the variant of the anastomosis formation. Now prospective randomized investigations, proving in a single-value way the advantage of certain procedure for the AC with the distal AA part hypoplasia, are absent.

The objective of prospective investigation was to compare two methods: modified reversed plasty (group I) and formation of «extended» anastomosis (group II).

Materials and methods. Results of the surgical treatment estimation in 64 patients, of early age, for the AC together with the distal AA part hypoplasia in $2011-2015$ yrs period are adduced.

Results. Analysis of remote results was conducted in $62(98.8 \%)$ patients in terms up to 48 mo. Summarized index of survival in terms up to 4 years have constituted $95.9 \%$ - in group I, and $95.6 \%$ - in group II. Diameter of aortal isthmus in the group II patients in remote period have constituted $(0.98 \pm 0.4) \mathrm{mm}$, and in group I $-(1.2 \pm 0.86) \mathrm{mm}$. In group I a recurrence of AC in remote period was absent in $98.2 \%$ of the patients, and in group II - in $96.3 \%$.

Conclusion. The method of modified reversed plasty, using the flap of left subclavian artery, is reproducible and secure, what was witnessed by the absence of fatal complications in early and remote postoperative period.

Keywords: aortal coarctation; hypoplasia of aortal arch; surgical correction; recurrent aortal coarctation.

Сочетание КА с гипоплазией ДА выявляют у $70 \%$ больных [1 - 4]. Наиболее часто (у 42\% больных) наблюдали гипоплазию дистальной части ДА, у 40\% - гипоплазию ее прокси- мальной части, у 18\% - гипоплазию перешейка аорты [5, 6]. Выбор оптимальной хирургической коррекции КА с гипоплазией ДА обсуждается [7]. В НМИЦ имени Е. Н. Мешалки- на разработана оригинальная технология, представляющая модифицированную реверсивную пластику дистальной части ДА с использованием лоскута левой подключичной 
артерии по Meier [8, 9]. Способ позволяет устранить КА, расширить гипоплазированную дистальную часть ДА, а также сохранить кровообращение по левой подключичной артерии. Предложенный метод более благоприятный для пациента, поскольку полностью сохраняется физиологическое кровообращение левой верхней конечности. Выполнение реконструкции позволяет полностью устранить препятствие кровотоку, а также избежать таких осложнений, как стил-синдром, что дает возможность применять методику не только у новорожденных, но и пациентов более старшего возраста $[1,2,10]$.

В исследовании представлена оценка отдаленных результатов применения модифицированной методики реверсивной пластики с использованием лоскута подключичной артерии с сохранением кровотока в левой верхней конечности и метода наложения расширенного анастомоза.

\section{МАТЕРИАЛЫ И МЕТОДЫ ИССЛЕДОВАНИЯ}

Использованы материалы НМИЦ имени академика Е. Н. Мешалкина и Центра детской кардиохирургии при НЦХ имени академика М. А. Топчибашева, основанные на результатах оценки хирургического лечения 64 пациентов раннего возраста по поводу КА в сочетании с гипоплазией дистальной части ДА в период с 2011 по 2015 г.

В зависимости от способа хирургической коррекции пациенты распределены на две группы.

I группа-пациенты, у которых коррекцию выполняли методом модифицированной реверсивной пластики с использованием лоскута левой подключичной артерии; II группа - пациенты, у которых формировали расширенный анастомоз.

Метод хирургической коррекции КА выбирали на основе данных инструментальных методов исследования.

Исследование сердечно-сосудистой системы включало клиническую оценку состояния больных, анамнез, ЭКГ, трансторакальную эхокардиографию. В целях уточне- ния анатомии, степени гипоплазии ДА до операции проводили мультиспиральную компьютерную томографию аорты с контрастированием.

Возраст больных в среднем $(2,1 \pm$ $1,02)$ мес - в I группе, $(2,7 \pm 1,8)$ мес - во II группе $(\mathrm{p}=0,45)$. Мальчиков было 67,1\%, девочек - 32,8\%. Площадь поверхности тела рассчитывали по формуле Mosteller, она составила $(0,22 \pm 0,13) \mathrm{M}^{2}-$ в I группе, $(0,27$ $\pm 0,19) \mathbf{м}^{2}-$ во II группе. По возрастным, гендерным и антропометрическим данным группы сопоставимы.

Изолированная КА с гипоплазией ДА диагностирована у 38 (59,3\%) больных, дефект межжелудочковой перегородки (ДМЖП) - у 26 (40,6\%). Размеры дефекта оценивали в сопоставлении с диаметром фиброзного кольца клапана аорты, при равном по размеру или большем его считали большим. У 14 (21,8\%) пациентов при ДМЖП выполнена паллиативная коррекция - устранение КА и операция Muller.

При оценке дистальной части ДА выявлена статистически значимая разница в группах, показатель дистальной части ДА в I группе составил в среднем $(0,55 \pm 0,1)$ мм, во II группе - $(0,7 \pm 0,1)$ мм $(p=0,001)$. Тубулярная гипоплазия ДА выявлена у 14 (43,7\%) больных I группы и 9 (28,1\%) - II группы.

Для оценки степени гипоплазии ДА выполняли расчеты Z-score, ocнованные на показателе Z-value величины, которую определяют как число стандартных (сигмальных) отклонений от нормального значения размера для данной площади поверхности тела. Значение Z-value менее - 2 свидетельствовало о выраженной гипоплазии ДА. При расчете Z-score у всех пациентов значение менее -2 свидетельствовало о выраженной гипоплазии расчетного участка ДА.

Все операции выполняли в плановом порядке, в условиях нормотермии, без искусственного кровообращения, по стандартному протоколу, со стандартным обеспечением, принятым в клинике.

Базу данных формировали в системе Microsoft Excel 2006. Статистическая обработка данных произ- ведена на персональном компьютере с применением пакета программ Statistica 6.0 (StatSoft, США).

Для составления представления о выборке использовали методы описательной статистики. Достоверность различий в сравниваемых группах (р) рассчитывали с использованием непараметрических критериев Mann-Whitney - в независимых группах и Wilcoxon - в зависимых, для категориальных данных - с помощью таблиц сопряжения с применением точного теста Фишеpa. Значимость различий в группах считали достоверной при $\mathrm{p}<0,05$. Кривые выживаемости построены на основании метода Kaplan-Meier. Для выявления предикторов, повлиявших на неблагоприятный результат, проведен однофакторный регрессионный анализ Кокса.

\section{РЕЗУЛЬТАТЫ}

При анализе отдаленных результатов исследования использовали данные 62 (98,8\%) пациентов в сроки наблюдения до 48 мес.

Общая госпитальная летальность составила 3,1\% (умерли 2 больных). В отдаленном периоде все пациенты живы. При динамическом наблюдении на основании полученных результатов построены кривые выживаемости для каждой группы. Ожидаемая кумулятивная выживаемость в сроки до 4 лет составила 95,9\% - в I группе, 95,6\% - во II группе.

В I группе в отдаленном периоде повторная КА с пиковым градиентом 34 мм рт. ст. возникла у 1 больного, во II группе - у 2.

Проведена оценка предикторов, влияющих на сроки до повторного возникновения КА в отдаленном периоде. Методы коррекции КА не были предикторами повторной КА (р = $0,86)$; статистически достоверными предикторами были форма ДА и пиковый градиент на уровне перешейка аорты.

В отдаленном периоде повторной КА не было у 98,2\% пациентов I группы и 96,3\% - II группы (p=0,49).

В отдаленном послеоперационном периоде выявлены достоверные различия таких показателей: диаметр перешейка аорты во II группе - $(0,98 \pm 0,4)$ мм, в I группе - $(1,2$ 
$\pm 0,86)$ мм ( $=0,003)$; градиент давления на уровне перешейка аорты в I группе-(12,2 $\pm 1,06)$ мм рт. ст., во II группе $-(15,5 \pm 1,89)$ мм рт. ст. $(\mathrm{p}=$ 0,002); гипертрофия миокарда левого желудочка (ЛЖ) во II группе - у 10 (40\%) больных, в I группе - у 2 (8\%).

Также оценивали геометрическую форму ДА на основе данных Р. Ou и соавторов [11, 12]. Авторы предложили классификацию ДА после хирургической коррекции КА. В зависимости от угла между восходящей и нисходящей частями аорты выделены три типа ДА: готическая (угловая), амбразурная (прямоугольная) и романская. Авторы предположили, что форма ДА является одним из факторов риска возникновения артериальной гипертензии в отдаленном периоде.

Готическая форма ДА преобладала у 25,8\% пациентов II группы (p = 0,003), романская - у 64,5\% пациентов I группы (p=0,02); амбразурную форму ДА наблюдали одинаково часто $(\mathrm{p}=0,31)$.

По данным корреляционного анализа, для выявления связи таких показателей, как гипертрофия миокарда ЛЖ и форма ДА, коэффициент корреляции Спирмена составил $\mathrm{r}=$ $-0,42(p=0,07)$. Таким образом, форма ДА может влиять на степень гипертрофии миокарда Лж.

\section{ОБСУЖДЕНИЕ}

Общая госпитальная летальность составила 3,1\%, что соответствует этому показателю у таких больных. Основной причиной смерти больных были пневмония, сепсис, что соответствует основным причинам, по данным литературы [13 - 15].

Статистически достоверных различий частоты повторной КА в группах не было.

При оценке геометрической формы ДА P. Ou и коллеги наблюдали патологические состояния, в частности, гипертрофию миокарда Лж, артериальную гипертензию у пациентов после успешной коррекции КА $[11,12]$. У пациентов при готической форме ДА отмечена значительно более высокая частота сосудистой дисфункции, чем при романской форме и в контрольной группе. При этом у таких пациентов не было признаков повторной КА. Мы также провели корреляционный анализ таких показателей, как гипертрофия миокарда ЛЖ и форма ДА, установлена положительная корреляционная связь $(\mathrm{p}=0,07)$, что соответствует показателям, представленным другими исследователями $[11,12]$.

При оценке гемодинамики в ДА после коррекции КА отмечено, что при романской форме ДА клинические результаты лучше, чем при готической и амбразурной [16]. В нашем исследовании готическую форму ДА выявляли только во II группе, романскую - в I группе (у 20 пациентов). Таким образом, при осуществлении модифицированной реверсивной пластики с использованием лоскута левой подключичной артерии можно смоделировать форму ДА, приближенную к естественной [3].

\section{выводы}

1. Метод модифицированной реверсивной пластики с использованием лоскута левой подключичной артерии воспроизводимый и безопасный, о чем свидетельствовало отсутствие фатальных осложнений как в раннем, так и отдаленном периоде после выполнения вмешательства.

2. Даже при отсутствии анатомических признаков повторной КА у 25\% пациентов II группы сохранялась артериальная гипертензия (в I группе - у 9,6\%), гипертрофию ЛЖ наблюдали во II группе - у 40\%, в I группе - у 8\%. Таким образом, у 33\% пациентов, у которых для коррекции КА формировали расширенный анастомоз, радикальная в анатомическом смысле операция не является радикальной в физиологическом и функциональном смысле.

3. Метод модифицированной реверсивной пластики с использованием лоскута левой подключичной артерии позволяет более чем у 67\% пациентов смоделировать форму ДА, приближенную к естественной.

\section{REFERENCES}

1. Sinelnikov YuS, Gorbatih YuN, Ivantsov SM. Surgical treatment of coarctation of the aorta with hypoplasia of the distal portion of the aortic arch in newborns. Pathology of circulation and cardiac surgery. 2011;(3): 9-12. [In Russian].

2. Sinelnikov YuS, Kshanovskaya MS, Gorbatih AV, Ivantsov SM. Hypoplasia of the aortic arch. Ibid. 2013;(3): 68-72. [In Russian].

3. Soinov IA, Sinelnikov YuS, Gorbatih AV, Nicy NR, Ivantsov SM, Kornilov IA hypertension in patients after correction of coarctation and hypoplasia of the aortic arch. Ibid. 2015;(2):102-13. [In Russian].

4. Kornilov IA, Sinelnikov YuS, Soinov IA, Kshanovskaya MS, Ponomarev DN, Matyushov VN. Risk assessment renal and neurological complications in newborns after reconstruction of the aorta. Ibid. 2015;(1): 84-9. [In Russian].

5. Barreiro CJ, Ellison TA, Williams JA, Durr ML, Cameron DE, Vricella LA. Subclavian flap aortoplasty: still a safe, reproducible, and effective treatment for infant coarctation. Eur J Cardiothorac Surg. 2007;31:649 -53. DOI:10.1016/j.ejcts.2006.12.038

6. Burch PT, Cowley CG, Holubkov R, Null D, Lambert LM, Kouretas PC, Hawkins JA. Coarctation repair in neonates and young infants: is small size or low weight still a risk factor? J Thorac Cardiovasc Surg. 2009; 138:547-52. doi: 10.1016/j.jtcvs.2009.04.046. Epub 2009 Jul 1.

7. Clarkson PM, Nicholson MR, Barratt-Boyes BG, Neutze JM, Whit- lock RM. Results after repair of coarctation of the aorta beyond infancy: a 10 to 28 year follow-up with particular reference to late systemic hypertension .Am J Cardiol. 2000;51:1481-8

8. Fruh S, Knirsch W, Dodge-Khatami A, Dave H, Pretre R, Kretschmar $O$. Comparison of surgical and interventional therapy of native and recurrent aortic coarctation regarding different age groups during childhood. Eur J Cardiothorac Surg 2011;39:898-904. doi: 10.1016/j.ejcts.2010.09.048. Epub 2010 Dec 18.

9. Wright GE, Nowak CA, Goldberg CS, Ohye RG, Bove EL, Rocchini AP. Extended Resection and End-to-End Anastomosis for Aortic Coarctation in Infants: Results of a Tailored Surgical Approach. Ann Thorac Surg 2005;80:1453-9.

10. McElhinney DB, Yang SG, Hogarty AN, Rychik J, Gleason $\mathrm{MM}$, Zachary $\mathrm{CH}$, et al. Recurrent arch obstruction after repair of isolated coarctation of the aorta in neonates and young infants: is low weight a risk factor? J Thorac Cardiovasc Surg. 2001;122(5):88390. DOI:10.1067/mtc.2001.116316

11. Meier MA, Lucchese FA, Jazbik W, Nesralla IA, Mendonca JT. A new technique for repair of aortic coarctation. Subclavian flap aortoplasty with preservation of arterial blood flow to the left arm. J Thorac Cardiovasc Surg.1986;92:1005-12. PMID:3784584

12. Pandey R, Jackson M, Ajab S, Gladman G, Pozzi M. Subclavian flap repair: review of 399 patients at median follow-up of fourteen 
years. Ann Thorac Surg. 2006;81:1420-8. DOI:10.1016/j.athoracsur.2005.08.070.

13. Simpson IA, Sahn DJ, Valdes-Cruz LM, Chung KJ, Sherman FS, Swensson RE. Color Doppler flowapping in patients with coarctation of the aorta: new observations and improved evaluation with color flow diameter and proximal acceleration as predictors of severity. Circulation.1988;77:736-44.

14. Olivieri LJ, de Zélicourt DA, Haggerty CM, Ratnayaka K, Cross RR, Yoganathan AP. Hemodynamic Modeling of Surgically Repaired Coarctation of the Aorta. Cardiovasc Eng Technol. 2011;2(4):28895. DOI:10.1007/s13239-011-0059-1
15. Ou P, Bonnet D, Auriacombe L, Pedroni E, Balleux F, Sidi D, et al. Late systemic hypertension and aortic arch geometry after successful repair of coarctation of the aorta. Eur Heart J. 2004; 25:1853-9. DOI:10.1016/j.ehj.2004.07.021.

16. Ou P, Celermajer DS, Raisky O, Jolivet O, Buyens F, Herment A et al. Angular (gothic) aortic arch leads to enhanced systolic wave reflection, central aortic stiffness, and increased left ventricular mass late after aortic coarctation repair: evaluation with magnetic resonance flow mapping. J Thorac Cardiovasc Surg. 2008;135(1):62-8. DOI:10.1016/j.jtcvs.2007.03.05. 